\title{
Multivoice Littlewood-Paley-Meyer Wavelets and Diagonal Dominated Pseudodifferential Operators
}

\section{Claudio Albanese}

Institut für Theoretische Physik, ETH-Hönggerberg, CH-8093 Zürich, Switzerland

Received: 27 January 1993/in revised form: 31 March 1993

\begin{abstract}
Given a pseudodifferential operator $\varepsilon(p)$ satisfying certain growth and smoothness conditions in momentum space, we construct a wavelet basis of $L^{2}\left(\mathbb{R}^{d}\right)$ in which the corresponding matrix is diagonal dominated with arbitrarily small prefactor.
\end{abstract}

\section{Introduction}

Problems arising in several branches of mathematical physics including quantum field theory, fluid dynamics and semiclassical analysis require some sort of multiscale analysis. The techniques based on the tree expansion in quantum field theory and on pseudodifferential calculus have recently been complemented by a new tool: the wavelet bases of $L^{2}\left(\mathbb{R}^{d}\right)$ discovered by Meyer, Lemarié, Daubechies, Mallat and others. We refer to Meyer's books [M] for a review of these results and for further references.

The prototypical basis found by Meyer in 1988 is given in dimension one by a family of functions $\psi_{x}(\xi) \in L^{2}(\mathbb{R}), x=(s(x), \xi(x)), s(x) \in \mathbb{Z}$ and $\xi(x) \in 2^{-s(x)} \mathbb{Z}$, which is generated by one "mother" function $\psi(\xi) \in L^{2}(\mathbb{R})$ so that

$$
\psi_{x}(\xi)=2^{\frac{s(x)}{2}} \psi\left(2^{s(x)}(\xi-\xi(x))\right)
$$

and

$$
\operatorname{supp} \hat{\psi}=\left\{p \in \mathbb{R}: \frac{\pi}{3} \leqq|p| \leqq \frac{4 \pi}{3}\right\}
$$

Meyer's wavelets also have a "father" $\phi(\xi)$ in terms of which $\psi(\xi)$ is defined and which helps to generate higher dimensional wavelets by the method of tensor products. In Sect. 2, we give a selfcontained review of these constructions. This particular basis has been named by Meyer after Littlewood and Paley. We find it thus natural to call these basis functions "LPM-wavelets." 
Our goal in this paper is to refine Meyer's construction of LPM wavelets in order to obtain wavelet bases with better localization properties in momentum space. The following specific question arose in an attempt to understand the Schwinger-Englert semiclassical expansions [E] within a rigorous framework [A], see also [FS] for a review of the rigorous results: given a pseudodifferential operator $\varepsilon(p)$ in a class defined below whose prototype is $p^{2}$, the problem is to construct a wavelet basis for which the matrix

$$
\varepsilon(x \mid y)=(2 \pi)^{-d} \int d p \varepsilon(p) \overline{\hat{\psi}_{x}(p)} \hat{\psi}_{y}(p)
$$

is diagonal dominated, i.e. is such that

$$
\sum_{x \neq y}|\varepsilon(x \mid y)|<c \cdot \varepsilon(x \mid x)
$$

for some $c<1$ and all $x$. Meyer's wavelets do not have this property for all functions $\varepsilon(p)$. In fact, even if $\varepsilon(p)$ is $C^{\infty}$, there is no way to squeeze the support of $\hat{\psi}$ to a set of arbitrarily short diameter if we also insist on having only one mother $\psi$. In order to refine the partition of momentum space, we resort to wavelet bases forming "polygamic families" which are generated by $2^{n}\left(2^{d}-1\right)$ father functions $\phi_{\alpha}(\xi)$ and as many mother functions $\psi_{\alpha}(\xi)$ with $n \geqq 1$. In the general $d$-dimensional case, this basis has the form $\left\{\psi_{x}\right\}_{x \in \Omega_{n}}$, where the index set $\Omega_{n}$ is

$$
\Omega_{n}=\left\{x=(\alpha(x), s(x), \xi(x)) ; \alpha(x)=1, \ldots 2^{n}\left(2^{d}-1\right), s(x) \in \mathbb{Z}, \xi(x) \in 2^{-s(x)} \mathbb{Z}^{d}\right\},
$$

and the functions $\psi_{x}(\xi)$ have the form

$$
\psi_{x}(\xi)=2^{\frac{d}{2} s(x)} \psi_{\alpha(x)}\left(2^{s(x)}(\xi-\xi(x))\right) .
$$

We have

Theorem. In all dimensions $d \geqq 1$ and for all integers $N, n \geqq 1$, there is a basis of $L^{2}\left(\mathbb{R}^{d}\right)$ of the form $\left\{\psi_{x}\right\}_{x \in \Omega_{n}}$, where $\Omega_{n}$ and $\psi_{x}$ are given above and we have:

(i) For all $x \in \Omega_{n}$, supp $\hat{\psi}_{x}$ is invariant under reflection with respect to the coordinate planes of $\mathbb{R}^{d}$ and we have

$$
\operatorname{supp} \hat{\psi}_{x} \cap \mathbb{R}_{+}^{d}=Q_{n}(x) \cap \mathbb{R}_{+}^{d},
$$

where $\mathbb{R}^{d}=\left\{\left(p_{1}, \ldots p_{d}\right): j \geqq 0 \forall j=1, \ldots d\right\}$ and $Q_{n}(x)$ is a cube in $\mathbb{R}^{d}$ whose side has length $2^{1-n} \pi$;

(ii) If $x_{1}, x_{2} \in \Omega_{n}$ are such that

$$
Q\left(x_{1}\right) \cap Q\left(x_{2}\right) \neq \emptyset,
$$

then $\left|s\left(x_{1}\right)-s\left(x_{2}\right)\right| \leqq 1$;

(iii) For all multiindices $m=\left(m_{1} \ldots m_{d}\right)$ such that $|m| \leqq N$ and all integers $\alpha \in$ $\left\{1, \ldots 2^{n}\left(2^{d}-1\right)\right\}$, we have

$$
\left|\frac{\partial^{m}}{\partial p^{m}} \hat{\psi}_{\alpha}(p)\right| \leqq c\left(2^{n+1} \pi\right)^{|m|},
$$

where $c$ is a constant dependent on $d$ and on $N$ but not on $n$.

We also have 
Corollary. Let $d \geqq 1$ and let $\varepsilon \in \mathscr{C}^{d+1}\left(\mathbb{R}^{d}\right)$ be a function which is symmetric under all reflections with respect to the coordinate planes and such that for all multiindices $m=\left(m_{1} \ldots m_{d}\right)$ with $|m| \leqq d+1$ we have

$$
\left|p^{m} \frac{\partial^{|m|}}{\partial p^{m}} \varepsilon(p)\right| \leqq c \cdot \varepsilon(p)
$$

for some constant $c$. Then, if $n \geqq 1$ and $\left\{\psi_{x}\right\}_{x \in \Omega_{n}}$ is the wavelet basis in the theorem above corresponding to $N=d+1$, we have

$$
\sum_{x \neq y} \mid \varepsilon(x \mid y) \leqq c \cdot 2^{-(d+1)(n-1)} \varepsilon(x \mid x)
$$

for all $x \in \Omega_{n}$, where $c$ is a constant dependent on $d$ but not on $n$. In particular, if $n$ is large enough then the matrix $\varepsilon(x \mid y)$ is diagonal dominated.

\section{Littlewood-Paley-Meyer (LPM) Wavelets}

Here we review Meyer's construction of LPM wavelets.

Let $\phi \in L^{2}(\mathbb{R})$ be a real valued function whose Fourier transform has the following properties:

$$
\begin{aligned}
& \hat{\phi}(p)=1 \forall|p| \leqq \pi-\delta_{0}, \\
& 0<\hat{\phi}(p)<1 \forall \pi-\delta_{0}<|p| \leqq \pi+\delta_{0}, \\
& \hat{\phi}(p)=0 \forall|p| \geqq \pi+\delta_{0}, \\
& \hat{\phi}(p)^{2}+\hat{\phi}(2 \pi-p)^{2}=1 \quad \forall 0 \leqq p \leqq 2 \pi,
\end{aligned}
$$

where $\delta_{0} \in(0, \pi)$ is an adjustable parameter that we fix below. Let us remark that, as a consequence of (2.1)-(2.4), we have

$$
\sum_{k \in \mathbb{Z}} \hat{\phi}(p+2 \pi k)^{2}=1
$$

Let $L_{\text {per }}^{2}(2 \pi)$ be the Hilbert space of the functions $m \in L_{\text {loc }}^{2}(\mathbb{R})$ of period $2 \pi$ with the following scalar product:

$$
\left(m_{1}, m_{2}\right)=\int_{0}^{2 \pi} d p m_{1}(p) m_{2}(p)
$$

and let $V_{s}, s \in \mathbb{Z}$, be the subspace of $L^{2}(\mathbb{R})$ of the functions whose Fourier transform is of the form

$$
m\left(2^{-s} p\right) \hat{\phi}\left(2^{-s} p\right)
$$

with $m \in L_{\text {per }}^{2}(2 \pi)$. If $m_{1}, m_{2} \in L_{\text {per }}^{2}(2 \pi)$, then thanks to (2.5) we have

$$
\int_{-\infty}^{\infty} d p \overline{m_{1}\left(2^{-s} p\right)} m_{2}\left(2^{-s} p\right) \hat{\phi}\left(2^{-s} p\right)^{2}=2^{s} \int_{0}^{2 \pi} d p \overline{m_{1}(p)} m_{2}(p) .
$$


In particular, for every fixed scale $s \in \mathbb{Z}$, the functions $\phi_{x}(\xi), x \in \Omega_{1}, s(x)=s$, such that

$$
\hat{\phi}_{x}(p)=2^{-\frac{1}{2} s(x)} \exp (i \cdot \xi(x) p) \hat{\phi}\left(2^{-s(x)} p\right)
$$

form an orthonormal basis of $V_{s}$. Let us notice that we also have

$$
\phi_{x}(\xi)=2^{\frac{1}{2} s(x)} \phi\left(2^{s(x)}(\xi-\xi(x))\right) .
$$

Lemma 2.1. (Meyer). For all $s \in \mathbb{Z}$, we have

(i) $\phi_{x}(\xi), x \in \Omega_{1}, s(x)=s$, is an orthonormal basis of $V_{s}$;

(ii) $V_{s} \subset V_{s+1}$;

(iii) $\bigcap_{s \in \mathbb{Z}} V_{s}=\{0\}$ and $\overline{\left(\bigcup_{s \in \mathbb{Z}} V_{s}\right)}=L^{2}(\mathbb{R})$;

(iv) $f(\xi) \in V_{s} \Leftrightarrow f(2 \xi) \in V_{s+1}$;

(v) For all $\xi_{0} \in 2^{-s} \mathbb{Z}$, if $f(\xi) \in V_{s}$, then $f\left(\xi-\xi_{0}\right) \in V_{s}$.

Proof. Only (ii) requires a proof. We have to show that for all $m_{1} \in L_{\text {per }}^{2}(2 \pi)$ there exists an $m_{2} \in L_{\text {per }}^{2}(2 \pi)$ such that

$$
m_{1}\left(2^{-s} p\right) \hat{\phi}\left(2^{-s} p\right)=m_{2}\left(2^{-s-1} p\right) \hat{\phi}\left(2^{-s-1} p\right) .
$$

It suffices to consider the case $s=-1$. One can set $m_{2}(p)=0$ for $|p| \geqq \frac{1}{2}\left(\pi+\delta_{0}\right)$ because in this case $\hat{\phi}(2 p)=0$. On the other hand, if $|p| \leqq \frac{1}{2}\left(\pi+\delta_{0}\right)$, we have $\hat{\phi}(p)=1$ so that

$$
m_{2}(p)=m_{1}(2 p) \hat{\phi}(2 p)
$$

Q.E.D.

A sequence of subspaces $V_{s}, s \in \mathbb{Z}$, of $L^{2}(\mathbb{R})$ with the properties above is said to provide a multiscale decomposition of $L^{2}(\mathbb{R})$.

Let $W_{s}$ be the orthogonal complement of $V_{s}$ in $V_{s+1}$, so that we have $V_{s+1}=V_{s} \oplus W_{s}$. Thanks to Lemma 2.1, we ahve

$$
L^{2}(\mathbb{R})=\bigoplus_{s \in \mathbb{Z}} W_{s}
$$

We also have

Lemma 2.2. (Meyer). For all $s \in \mathbb{Z}$, the space $\mathscr{F} W_{s}$ is spanned by the functions of the form

$$
m\left(2^{-s} p\right) \hat{\psi}\left(2^{-s} p\right)
$$

with $m \in L_{\mathrm{per}}^{2}(2 \pi)$ and

$$
\hat{\psi}(p)=e^{-\frac{i}{2} p}\left(\hat{\phi}\left(\frac{1}{2} p\right)^{2}-\hat{\phi}(p)^{2}\right)^{1 / 2} .
$$

Moreover the application $\mathscr{R}: L_{\mathrm{per}}^{2}(2 \pi) \rightarrow \mathscr{F} W_{s}$ such that

$$
\mathscr{R}(m)(p)=2^{-\frac{s}{2}} \cdot m\left(2^{-s} p\right) \cdot \hat{\psi}\left(2^{-s} p\right)
$$

is an isometry. 
Proof. It suffices to consider the case $s=-1$. Let us introduce the following $2 \pi$-periodic function:

$$
m_{0}(p)=\sum_{\xi_{0} \in \mathbb{Z}} \frac{1}{2} e^{i p \xi_{0}} \int_{-\infty}^{\infty} d \xi \overline{\phi\left(\xi+\xi_{0}\right)} \phi\left(\frac{1}{2} \xi\right) .
$$

Since $V_{-1} \subset V_{0}$, we can write

$$
\frac{1}{2} \phi\left(\frac{1}{2} \xi\right)=\sum_{\xi_{0} \in \mathbb{Z}}\left[\frac{1}{2} \int_{-\infty}^{\infty} d \xi \overline{\phi\left(\xi+\xi_{0}\right)} \phi\left(\frac{1}{2} \xi\right)\right] \phi\left(\xi+\xi_{0}\right) .
$$

By taking the Fourier transform of both members, we find

$$
\hat{\phi}(2 p)=m_{0}(p) \hat{\phi}(p) \text {. }
$$

Moreover, thanks to the basic equality (2.5), we have

$$
\begin{aligned}
1 & =\sum_{k \in \mathbb{Z}} \hat{\phi}(2 p+2 \pi k)^{2}=\sum_{k \in \mathbb{Z}} m_{0}(p+\pi k)^{2} \hat{\phi}(p+\pi k)^{2} \\
& =\sum_{k \in \mathbb{Z}}\left[m_{0}(p+2 \pi k)^{2} \hat{\phi}(p+2 \pi k)^{2}+m_{0}(p+\pi(2 k+1))^{2} \hat{\phi}(p+\pi(2 k+1))^{2}\right] \\
& =m_{0}(p)^{2}+m_{0}(p+\pi)^{2} .
\end{aligned}
$$

We have

$$
V_{-1}=\left\{m(2 p) m_{0}(p) \hat{\phi}(p), m \in L_{\text {per }}^{2}(2 \pi)\right\}
$$

and $W_{-1}$ is the space of the functions of the form $l(p) \hat{\phi}(p)$ with $l \in L_{\mathrm{per}}^{2}(2 \pi)$ such that

$$
0=\int_{-\infty}^{\infty} d p \overline{l(p)} m(2 p) m_{0}(p) \hat{\phi}(p)^{2}=\int_{0}^{2 \pi} d p \overline{l(p)} m(2 p) m_{0}(p)
$$

for all $m(p) \in L_{\text {per }}^{2}(2 \pi)$. Hence, $\overline{l(p)} m_{0}(p)$ has a Fourier series of the form

$$
\overline{l(p)} m_{0}(p)=\sum_{\xi_{0} \in \mathbb{Z}} c\left(\xi_{0}\right) \exp \left(i\left(2 \xi_{0}+1\right) p\right)
$$

i.e. we have

$$
\overline{l(p)} m_{0}(p)+\overline{l(p+\pi)} m_{0}(p+\pi)=0 .
$$

Due to (2.17), (2.20) and to the symmetry property $\phi(\xi)=\phi(-\xi),\left(m_{0}(p)\right.$, $\left.m_{0}(p+\pi)\right)$ is a real vector of unit norm of $\mathbb{C}^{2}$. Hence, the vector $(l(p), l(p+\pi))$ must be proportional to the orthogonal vector $\left(m_{0}(p+\pi),-m_{0}(p)\right)$ for all $p$. We thus find

$$
l(p)=e^{-i p} m_{0}(p+\pi) m(2 p)
$$

for some $m \in L_{\text {per }}^{2}(2 \pi)$. Finally, thanks to (2.18) and (2.19), we have

$$
\begin{aligned}
l(p) \hat{\phi}(p) & =e^{-i p}\left(1-m_{0}(p)^{2}\right)^{1 / 2} \hat{\phi}(p) m(p) \\
& =e^{-i p}\left(1-\frac{\hat{\phi}(2 p)^{2}}{\hat{\phi}(p)^{2}}\right)^{1 / 2} \hat{\phi}(p) m(2 p) \\
& =e^{-i p}\left(\hat{\phi}(p)^{2}-\hat{\phi}(2 p)^{2}\right)^{1 / 2} m(2 p) .
\end{aligned}
$$


Finally, if $m \in L_{\text {per }}^{2}(2 \pi)$, thanks to (2.5) we have

$$
\begin{aligned}
\|\mathscr{R}(m)(p)\|_{2}^{2} & =2^{-s} \int_{-\infty}^{\infty} d p m\left(2^{-s} p\right)^{2}\left|\hat{\psi}\left(2^{-s} p\right)\right|^{2} \\
& =\int_{-\infty}^{\infty} d p m(p)^{2}\left(\hat{\phi}\left(\frac{p}{2}\right)^{2}-\hat{\phi}(p)^{2}\right)=\int_{0}^{2 \pi} d p m(p)^{2} .
\end{aligned}
$$

Q.E.D.

This completes the construction of LPM wavelets in the one dimensional case. In dimension $d \geqq 1$ we can also obtain LPM wavelets by using the method of tensor products described below in Sect. 4 .

\section{Decomposition of the Space $L_{\text {per }}^{2}(2 \pi)$}

Let us introduce a family of real valued functions $\theta_{0}(\delta ; p) \in L_{\text {per }}^{2}(2 \pi)$ parametrized by $\delta \in\left(0, \frac{\pi}{2}\right)$ and such that

$$
\begin{gathered}
\theta_{0}(\delta ; p)=\theta_{0}(\delta ;-p) \quad \forall p \in \mathbb{R}, \\
\theta_{0}(\delta ; p)=1 \quad \text { if } \frac{\pi}{2}+\delta \leqq p \leqq \frac{3 \pi}{2}-\delta, \\
0<\theta_{0}(\delta ; p)<1 \quad \text { if } \frac{3 \pi}{2}-\delta<p<\frac{3 \pi}{2}+\delta, \\
\theta_{0}(\delta ; p)^{2}+\theta_{0}(\delta ; p+\pi)^{2}=1 \quad \forall p \in \mathbb{R} .
\end{gathered}
$$

Let $\theta_{1}(\delta ; p)$ be the function such that

$$
\theta_{1}(\delta ; p)=e^{i p} \theta_{0}(\delta ; p+\pi)
$$

and let

$$
\mathscr{T}_{\delta}: L_{\text {per }}^{2}(\pi) \oplus L_{\text {per }}^{2}(\pi) \rightarrow L_{\text {per }}^{2}(2 \pi)
$$

be the operator defined in such a way that if $m_{0}, m_{1} \in L_{\text {per }}^{2}(\pi)$, then we have

$$
\mathscr{T}_{\delta}\left(m_{0}, m_{1}\right)(p)=m_{0}(p) \theta_{0}(\delta ; p)+m_{1}(p) \theta_{1}(\delta ; p) .
$$

Lemma 3.1. The operator $\mathscr{T}_{\delta}$ is an isometry, i.e.

$$
\left\|\mathscr{T}_{\delta}\left(m_{0}, m_{1}\right)\right\|_{L_{\mathrm{per}}^{2}(2 \pi)}^{2}=\left\|m_{0}\right\|_{L_{\mathrm{per}}^{2}(\pi)}^{2}+\left\|m_{1}\right\|_{L_{\mathrm{per}}^{2}(\pi)}^{2}
$$

for all $m_{0}, m_{1} \in L_{\text {per }}^{2}(\pi)$ and

$$
\mathscr{R a n} \mathscr{T}_{\delta}=L_{\text {per }}^{2}(2 \pi) \text {. }
$$

Proof. Let us start by proving that for all $m_{0}, m_{1} \in L_{\text {per }}^{2}(\pi)$, we have

$$
\begin{aligned}
\int_{0}^{2 \pi} d p\left(\overline{m_{0}(p)} \theta_{0}(\delta ; p)\right. & \left.+\overline{m_{1}(p)} \overline{\theta_{1}(\delta ; p)}\right)\left(m_{0}(p) \theta_{0}(\delta ; p)+m_{1}(p) \theta_{1}(\delta ; p)\right) \\
& =\int_{0}^{\pi} d p\left(\left|m_{0}(p)\right|^{2}+\left|m_{1}(p)\right|^{2}\right)
\end{aligned}
$$


By using (3.4), we find

$$
\begin{aligned}
& \int_{0}^{2 \pi} d p\left|m_{0}(p)\right|^{2} \theta_{0}(\delta ; p)^{2}=\int_{0}^{\pi} d p\left|m_{0}(p)\right|^{2}, \\
& \int_{0}^{2 \pi} d p\left|m_{1}(p)\right|^{2}\left|\theta_{1}(\delta ; p)\right|^{2}=\int_{0}^{\pi} d p\left|m_{1}(p)\right|^{2} .
\end{aligned}
$$

Moreover, we have

$$
\begin{aligned}
& \int_{0}^{2 \pi} d p \overline{m_{1}(p)} m_{0}(p) \overline{\theta_{1}(\delta ; p)} \theta_{0}(\delta ; p) \\
& \quad=\int_{0}^{2 \pi} d p \overline{m_{1}(p)} m_{0}(p) e^{-i p} \theta_{0}(\delta ; p) \theta_{0}(\delta ; p+\pi)=0
\end{aligned}
$$

because

$$
e^{-i(p+\pi)} \theta_{0}(\delta ; p+\pi) \theta_{0}(\delta ; p+2 \pi)=-e^{-i p} \theta_{0}(\delta ; p) \theta_{0}(\delta ; p+\pi) .
$$

Similarly, one can show that

$$
\int_{0}^{2 \pi} d p \overline{m_{0}(p)} m_{1}(p) \theta_{0}(\delta ; p) \theta_{1}(\delta ; p)=0 .
$$

It remains to prove that $\mathscr{R} a n \mathscr{T}_{\delta}=L_{\text {per }}^{2}(2 \pi)$. The functions of the form $m(p) \theta_{0}(\delta ; p)$ (resp. $m(p) \theta_{1}(\delta ; p)$ ) with $m \in L_{\text {per }}^{2}(\pi)$, span the subspace of $L_{\text {per }}^{2}(2 \pi)$ of the functions with support in $\left[\frac{\pi}{2}+\delta, \frac{3 \pi}{2}-\delta\right]+2 \pi \mathbb{Z}$ (resp. $\left.\left[-\frac{\pi}{2}+\delta,+\frac{\pi}{2}-\delta\right]+2 \pi \mathbb{Z}\right)$. Moreover, if $f \in L_{\text {per }}^{2}(2 \pi)$ is a function with support in $\left(\frac{\pi}{2}-\delta, \frac{\pi}{2}+\delta\right)+2 \pi \mathbb{Z}$, then there are two functions $m_{0}, m_{1} \in L_{\mathrm{per}}^{2}(\pi)$ with support in $\left(\frac{\pi}{2}-\delta, \frac{\pi}{2}+\delta\right)+\pi \mathbb{Z}$ such that

$$
m_{0}(p) \theta_{0}(\delta ; p)+m_{1}(p) \theta_{1}(\delta ; p)=\varepsilon(p)
$$

for all $p \in \mathbb{R}$. To find these functions, let us observe that for all $p \in$ $\left(\frac{\pi}{2}-\delta, \frac{\pi}{2}+\delta\right)+2 \pi \mathbb{Z}$, we must have

$$
\begin{aligned}
0 & =m_{0}(p+\pi) \theta_{0}(\delta ; p+\pi)+m_{1}(p+\pi) \theta_{1}(\delta ; p+\pi) \\
& =m_{0}(p) e^{-i p} \theta_{1}(\delta ; p)-m_{1}(p) e^{i p} \theta_{0}(\delta ; p)
\end{aligned}
$$

and hence

$$
m_{0}(p)=e^{2 i p} m_{1}(p) \theta_{0}(\delta ; p) \theta_{1}(\delta ; p)^{-1}
$$

For such values of $p$, we also have

$$
\begin{aligned}
f(p) & =m_{1}(p)\left[e^{2 i p} \theta_{0}(\delta ; p)^{2} \theta_{1}(\delta ; p)^{-1}+\theta_{1}(\delta ; p)\right] \\
& =m_{1}(p) \theta_{1}(\delta ; p)^{-1} e^{2 i p}\left[\theta_{0}(\delta ; p)^{2}+\theta_{0}(\delta ; p+\pi)^{2}\right] \\
& =m_{1}(p) \theta_{1}(\delta ; p)^{-1} e^{2 i p} .
\end{aligned}
$$


Hence, we find the following explicit expressions for $m_{0}$ and $m_{1}$ :

$$
\begin{gathered}
m_{1}(p)=f(p) \theta_{1}(\delta ; p) e^{-2 i p}+f(p+\pi) \theta_{1}(\delta ; p+\pi) e^{-2 i p} \\
m_{0}(p)=f(p) \theta_{0}(\delta ; p)+f(p+\pi) \theta_{0}(\delta ; p+\pi)
\end{gathered}
$$

Q.E.D. . . .

\section{4. $2^{n}$-voice LPM Wavelets}

In this section, by combining Meyer's construction of LPM wavelets and the decomposition of the space $L_{\text {per }}^{2}(2 \pi)$ given above, we construct multivoice LPM wavelets which have all the properties required by the theorem in Sect. 1 and by its corollary.

The decomposition of the space $L_{\text {per }}^{2}(2 \pi)$ provided by the operators $\mathscr{T}_{\delta}$ in (3.6) can be iterated. Namely, for all integers $n \geqq 1$ and $\delta \in\left(0,2^{-n} \pi\right)$ one can define an isometry

$$
\mathscr{T}_{\delta}^{(n)}: \bigoplus_{j=1} L_{2^{n}}^{2}\left(2^{-n} \pi\right) \rightarrow L_{\text {per }}^{2}(2 \pi)
$$

such that if $m_{\sigma} \in L_{\text {per }}^{2}\left(2^{1-n} \pi\right)$ is a family of functions labelled by elements $\sigma$ in the set

$$
s_{n}=\left\{\sigma=\left(\sigma_{1} \ldots \sigma_{n}\right) \mid \sigma_{m} \in\{0,1\} \forall m=1 \ldots n\right\}
$$

we have

$$
\mathscr{T}_{\delta}^{n}\left(m_{\sigma}\right)(p)=\sum_{\sigma} m_{\sigma}(p) \theta_{\sigma}(\delta ; p)
$$

Here, we set

$$
\theta_{\sigma}(\delta ; p)=\theta_{\sigma_{1}}(\delta ; p) \cdot \prod_{m=2, \quad} \theta_{1+\sigma_{m}+\sigma_{m-1}}\left(\delta_{m} ; 2^{m-1} p\right)
$$

where

$$
\delta_{m}=2^{m-1} \delta,
$$

and the sum $\left(1+\sigma_{m}+\sigma_{m-1}\right)$ is computed $\bmod 2$ and has values in $\{0,1\}$. With this definition of $\theta_{\sigma}$, we have

Lemma 4.1. For all fixed $n \geqq 1$ and all $\delta \in\left(0,2^{1-n} \pi\right)$, we have

$$
\theta_{\sigma}(\delta ;-p)=\overline{\theta_{\sigma}(\delta ; p)}
$$

and

$$
\operatorname{supp} \theta_{\sigma}(\delta ; \cdot) \cap[\pi-\delta, 2 \pi+\delta]=\pi+\sum_{m=1}^{n} \sigma_{m} 2^{-m} \pi+\left[-\delta, 2^{-n} \pi+\delta\right] .
$$

Proof. Equation (4.6) is obvious. If $n=1$, then (4.7) follows from (3.1) and (3.5). Otherwise, if $n \geqq 2,(4.7)$ can be proved by induction in $n$. In fact, let us suppose (4.7) holds for all $\sigma \in S_{n-1}$ and let us fix a $\delta \in\left(0,2^{-n} \pi\right)$ and a $\sigma \in s_{n}$. We have

$$
\theta_{\sigma}(\delta ; p)=\theta_{\sigma^{\prime}}(\delta ; p) \theta_{1+\sigma_{n}+\sigma_{n-1}}\left(\delta_{n} ; 2^{n-1} p\right),
$$


where $\sigma^{\prime}=\left(\sigma_{1} \ldots \sigma_{n-1}\right) \in S_{n-1}$. We also have

$$
\begin{aligned}
\operatorname{supp} & \theta_{1+\sigma_{n}+\sigma_{n-1}}\left(\delta_{n} ; 2^{n-1} p\right) \\
= & {\left[2^{-n} \pi-\delta, 3 \cdot 2^{-n} \pi+\delta\right]+\left(1+\sigma_{n}+\sigma_{n-1}\right) \cdot 2^{1-n} \pi+2^{2-n} \pi \mathbb{Z} } \\
= & {\left[2^{-n}\left(2 \sigma_{n}+3\right) \pi-\delta, 2^{-n}\left(2 \sigma_{n}+5\right) \pi+\delta\right]+\sigma_{n-1} \cdot 2^{1-n} \pi+2^{2-n} \pi \mathbb{Z} } \\
= & {\left[2^{-n}\left(2 \sigma_{n}-1\right) \pi-\delta, 2^{-n}\left(2 \sigma_{n}+1\right) \pi+\delta\right]+\pi } \\
& +\sum_{m=1}^{n-1} \sigma_{m} \cdot 2^{-m} \pi+2^{2-n} \pi \mathbb{Z} .
\end{aligned}
$$

Hence

$$
\begin{aligned}
\operatorname{supp} & \theta_{\sigma}(\delta ; \cdot) \cap[\pi-\delta, 2 \pi+\delta] \\
= & \left(\pi+\sum_{m=1}^{n-1} \sigma_{m} 2^{-m} \pi+\left[-\delta, 2^{-n+1} \pi+\delta\right]\right) \\
& \cap\left(\pi+\sum_{m=1}^{n-1} \sigma_{m} 2^{-m} \pi+\left[2^{-n}\left(2 \sigma_{n}-1\right) \pi-\delta,\right.\right. \\
= & \left.\left.\pi+\sum_{m=1}^{n-n}\left(2 \sigma_{n}+1\right) \pi+\delta\right]+2^{2-n} \pi \mathbb{Z}\right) \\
= & \pi+\sum_{m=1}^{n} \sigma_{m} 2^{-m} \pi+\left[2^{-n} \sigma_{n} \pi-\delta, 2^{-n}\left(\sigma_{n}+1\right) \pi+\delta\right]
\end{aligned}
$$

Q.E.D.

One can now construct $2^{n}$-voices LPM wavelets. The fathers $\phi_{\sigma}$ and the mothers $\psi_{\sigma}, \sigma \in s_{n}$, are defined as the functions such that

$$
\begin{aligned}
& \hat{\phi}_{\sigma}(p)=\theta_{\sigma}(\delta ; p) \hat{\phi}(p), \\
& \hat{\psi}_{\sigma}(p)=\theta_{\sigma}(\delta ; p) \hat{\psi}(p),
\end{aligned}
$$

where $\delta \in\left(0,2^{-n} \pi\right), \hat{\phi}(p)$ is a function satisfying (2.1)-(2.4), and, according to Lemma $2.1, \hat{\psi}(p)$ is given by

$$
\hat{\psi}(p)=e^{-\frac{i}{2} p}\left(\hat{\phi}\left(\frac{1}{2} p\right)^{2}-\hat{\phi}(p)^{2}\right)^{1 / 2}
$$

If we set

$$
\begin{aligned}
& \phi_{\sigma s \xi_{0}}(\xi)=2^{\frac{1}{2} s} \phi_{\sigma}\left(2^{s}\left(\xi-\xi_{0}\right)\right), \\
& \psi_{\sigma s \xi_{0}}(\xi)=2^{\frac{1}{2} s} \psi_{\sigma}\left(2^{s}\left(\xi-\xi_{0}\right)\right),
\end{aligned}
$$

then thanks to the preceding lemma, we have 
Lemma 4.2. For all $n \geqq 1, \delta \in\left(0,2^{-n} \pi\right)$ and $\sigma \in s_{n}$, there exist functions $\psi_{\sigma}(\xi), \phi_{\sigma}(\xi)$ such that

(i)

$$
\hat{\psi}_{\sigma}(p)=\overline{\hat{\psi}_{\sigma}(-p)}, \quad \hat{\phi}_{\sigma}(p)=\overline{\hat{\phi}_{\sigma}(-p)}
$$

(ii)

$$
\operatorname{supp} \hat{\psi}_{\sigma} \cap \mathbb{R}_{+}=\pi+\sum_{m=1}^{n} \sigma_{m} 2^{-m} \pi+\left[-\delta, 2^{-n} \pi+\delta\right]
$$

and

$$
\operatorname{supp} \hat{\phi}_{\sigma} \cap \mathbb{R}_{+}=\left(\sum_{m=1}^{n}\left(1-\sigma_{m}\right) 2^{-m} \pi+\left[-\delta, 2^{-n} \pi+\delta\right]\right) \cap \mathbb{R}_{+}
$$

(iii)

$$
\left\langle\phi_{\sigma s \xi_{0}} \mid \phi_{\sigma^{\prime} s \xi_{0}^{\prime}}\right\rangle=\delta_{\sigma \sigma^{\prime}} \delta_{\xi_{0} \xi_{0}^{\prime}}
$$

and

$$
\left\langle\psi_{\sigma s \xi_{0}} \mid \psi_{\sigma^{\prime} s^{\prime} \xi_{0}^{\prime}}\right\rangle=\delta_{\sigma \sigma^{\prime}} \delta_{s s^{\prime}} \delta_{\xi_{0} \xi_{0}^{\prime}}
$$

for all $\sigma, \sigma^{\prime} \in s_{n}, s, s^{\prime} \in \mathbb{Z}, \xi_{0} \in 2^{n-s} \mathbb{Z}, \xi_{0}^{\prime} \in 2^{n-s^{\prime}} \mathbb{Z}$;

(iv) The spaces

$$
V_{s}=\overline{\operatorname{span}\left\{\phi_{\text {os } \xi_{0}}, \sigma \in s_{n}, \xi_{0} \in 2^{n-s} \mathbb{Z}\right\}},
$$

$s \in \mathbb{Z}$, give a multiscale decomposition of $L^{2}(\mathbb{R})$;

(v) If $s \in \mathbb{Z}$ and

$$
W_{s}=\overline{\operatorname{span}\left\{\psi_{\sigma s \xi_{0}}, \sigma \in s_{n}, \xi_{0} \in 2^{n-s} \mathbb{Z}\right\}},
$$

then

$$
V_{s+1}=W_{s} \oplus V_{s} .
$$

(Remark. These spaces are the same ones defined in Sect. 2.) In particular, we have

$$
L^{2}(\mathbb{R})=\bigoplus_{s \in \mathbb{Z}} W_{s}
$$

and $\psi_{\sigma s \xi_{0}}\left\{\sigma \in s_{n}, s \in \mathbb{Z}, \xi_{0} \in 2^{n-s} \mathbb{Z}\right\}$, is an orthonormal basis of $L^{2}(\mathbb{R})$;

(vi) For all integers $m \in[1, N]$ and all $\delta \in\left(0,2^{-n} \pi\right)$ we have

$$
\begin{gathered}
\sup _{p}\left|\frac{d^{m}}{d p^{m}} \hat{\phi}_{\sigma}(p)\right| \leqq c \delta^{-m}, \\
\sup _{p}\left|\frac{d^{m}}{d p^{m}} \hat{\psi}_{\sigma}(p)\right| \leqq c \delta^{-m},
\end{gathered}
$$

where $c$ is a constant independent of both $\delta$ and $n$. 
Proof. Equation (4.19) follows from (2.8) and (3.13), while (4.20) can be derived from Lemma 2.1 (ii) and from (2.2), (2.27) and (3.13). Only the last statement requires a proof. Let us consider the following function:

$$
\mu(p)= \begin{cases}0 & \text { if } p \leqq 0 \\ \mu_{0} \int_{0}^{p} d p^{\prime} p^{N-1}\left(p^{\prime}-\delta\right)^{N-1} & \text { if } 0 \leqq p \leqq \frac{1}{2} \delta, \\ 1 / 2 & \text { if } p>\frac{1}{2} \delta\end{cases}
$$

where $\mu_{0}$ is the following normalization constant:

$$
\mu_{0} \equiv \frac{1}{2}\left(\int_{0}^{\frac{\delta}{2}} d p^{\prime} p^{\prime N-1}\left(p^{\prime}-\delta\right)^{N-1}\right)^{-1} \leqq \text { const. } \delta^{-2 N+1} .
$$

If $m$ is an integer in $[1, N]$ and $p \in\left[0, \frac{1}{2} \delta\right]$, we have

$$
\frac{d^{m}}{d p^{m}} \mu(p)=\mu_{0}\left|\frac{d^{m-1}}{d p^{m-1}} p^{N-1}(p-\delta)^{N-1}\right| \leqq \text { const. } \delta^{-m}
$$

and

$$
\frac{d^{m}}{d p^{m}} \sqrt{1-\mu(p)^{2}} \leqq \text { const. } \delta^{-m} .
$$

Let us define $\hat{\phi}(p) \in L^{2}(\mathbb{R})$ to be the function satisfying (2.1-4) with $\delta_{0}=\delta$ and such that

$$
\begin{aligned}
& \hat{\phi}(p)=\mu(p+\pi+\delta) \text { if }-\pi-\delta \leqq p \leqq-\pi, \\
& \hat{\phi}(p)=\sqrt{1-\mu(p+\pi)^{2}} \text { if }-\pi \leqq p \leqq-\pi+\delta .
\end{aligned}
$$

We clearly have

$$
\left|\frac{d^{m}}{d p^{m}} \hat{\phi}(p)\right| \leqq \text { const. } \delta^{-m}
$$

for all $1 \leqq m \leqq N$. Similarly, if $\theta(\delta ; p)$ is a function satisfying (3.1)-(3.4) and such that

$$
\begin{aligned}
& \theta_{0}(\delta ; p)=\mu\left(p-\frac{\pi}{2}+\delta\right) \text { if } \frac{\pi}{2}-\delta \leqq p \leqq \frac{\pi}{2}, \\
& \theta_{0}(\delta ; p)=\sqrt{1-\mu\left(p-\frac{\pi}{2}\right)^{2}} \quad \text { if } \frac{\pi}{2} \leqq p \leqq \frac{\pi}{2}+\delta,
\end{aligned}
$$

we have

$$
\frac{d^{m}}{d p^{m}} \theta_{0}(\delta ; p) \leqq \text { const. } \delta^{-m}
$$

for all $1 \leqq m \leqq N$. Thanks to (4.4), we also have

$$
\frac{d^{m}}{d p^{m}} \theta_{\sigma}(\delta ; p) \leqq \text { const. } \delta^{-m}
$$

for all $n \geqq 1$ and all $\sigma \in s_{n}$. Hence, (4.25) and (4.26) are also valid. Q.E.D. 
Once one knows a family of fathers $\phi_{\sigma}(\xi)$ and mothers $\psi_{\sigma}(\xi), \sigma \in s_{n}$, of one dimensional, $2^{n}$-voices LPM wavelets, one can construct similar wavelet bases in any dimension $d \geqq 2$ by the method of tensor products. In fact, let us consider the subspaces $\mathscr{V}_{s}$ and $\mathscr{W}_{s}, s \in \mathbb{Z}$, of $L^{2}\left(\mathbb{R}^{d}\right)$ such that

$$
\mathscr{V}_{s}=\bigotimes_{j=1, \quad{ }_{d}} V_{s}, \quad \mathscr{V}_{s+1}=\mathscr{V}_{s} \oplus \mathscr{W}_{s}
$$

We have

$$
\mathscr{W}_{s}=\bigoplus_{\sigma \in s_{n}, \tau \in t_{d}} \bigoplus_{j=1, \quad d} \mathscr{F}_{\sigma s}^{\tau_{j}}
$$

where

$$
t_{d}=\left\{\tau=\left(\tau_{1} \ldots \tau_{d}\right): \tau_{j} \in\{0,1\}, j=1 \ldots d, \text { and }\left(\tau_{1} \ldots \tau_{d}\right) \neq(0, \ldots 0)\right\}
$$

and

$$
\begin{aligned}
& F_{\sigma s}^{0}=\overline{\operatorname{span}\left\{\phi_{\sigma s \xi_{0}}, \xi_{0} \in 2^{n-s} \mathbb{Z}\right\}}, \\
& F_{\sigma s}^{1}=\overline{\operatorname{span}\left\{\psi_{\sigma s \xi_{0}}, \xi_{0} \in 2^{n-s} \mathbb{Z}\right\}} .
\end{aligned}
$$

If $\alpha=(\sigma, \tau) \in s_{n} \times t_{d}$, let us introduce the functions

$$
\psi_{\alpha}(\xi)=\prod_{j=1} f_{\sigma}^{\tau_{j}}\left(\xi_{j}\right)
$$

where $\xi=\left(\xi \ldots \xi_{d}\right) \in \mathbb{R}^{d}$ and

$$
f_{\sigma}^{0}\left(\xi_{j}\right)=\phi_{\sigma}\left(\xi_{j}\right), \quad f_{\sigma}^{1}\left(\xi_{j}\right)=\psi_{\sigma}\left(\xi_{j}\right) .
$$

Let $\Omega_{n}$ be the set in (1.5), i.e.

$$
\Omega_{n}=\left\{(\alpha(x), s(x), \xi(x)), \alpha(x) \in s_{n} \times t_{d}, s(x) \in \mathbb{Z}, \xi(x) \in 2^{n-s} \mathbb{Z}^{d}\right\} .
$$

If $x \in \Omega_{n}$, let $\psi_{x}$ be the function such that

$$
\psi_{x}(\xi)=2^{\frac{d}{2} s(x)} \psi_{\alpha(x)}\left(2^{s(x)}(\xi-\xi(x))\right) .
$$

Then, for all $x_{1}, x_{2} \in \Omega_{n}$ we have

$$
\left\langle\psi_{x_{1}} \mid \psi_{x_{2}}\right\rangle=\delta_{x_{1} x_{2}}
$$

and $\left\{\psi_{x}\right\}_{x \in \Omega_{n}}$ is an orthonormal basis of $L^{2}\left(\mathbb{R}^{d}\right)$.

It is easy to see that if the functions $\phi_{\sigma}$ and $\psi_{\sigma}$ are chosen so that they have all the properties in Lemma 4.2 for $\delta=2^{-n-1} \pi$, then the basis $\left\{\psi_{x}\right\}_{x \in \Omega_{n}}$ has all the properties required in the theorem of Sect. 1. In fact, supp $\hat{\psi}_{x}$ is invariant under all reflections with respect to one of the coordinate planes of $\mathbb{R}^{d}$. Moreover, we have

$$
\operatorname{supp} \hat{\psi}_{x} \cap \mathbb{R}_{+}^{d}=Q(x) \cap \mathbb{R}_{+}^{d},
$$

where $Q(x)$ is a cube in $\mathbb{R}^{d}$ whose side has length $2^{1-n-s(x)} \pi$. The center $p(x)=\left(p_{1}(x), \ldots p_{d}(x)\right) \in \mathbb{R}^{d}$ of $Q(x)$ is such that, for all $j=1, \ldots d$, we have

$$
p_{j}(x)=2^{s} \pi\left[2^{-n-1}+\sum_{m=1}^{n}\left(1-\sigma_{m}\right) 2^{-m}\right]
$$


in case $\tau_{j}(x)=0$ and

$$
p_{j}(x)=2^{s} \pi\left[1+2^{-n-1}+\sum_{m=1}^{n} \sigma_{m} 2^{-m}\right]
$$

if $\tau_{j}(x)=1$. In particular, for all $x \in \Omega_{n}$ there are no more than $\left(2^{d}+2 d-2\right)$ wavelets $y \in \Omega_{n}$ with $\xi(x)=\xi(y)$ such that

$$
Q(x) \cap Q(y) \neq \emptyset,
$$

up to irrelevant boundaries of zero Lebesgue measure. Finally, let us pass to the proof of the corollary in the introduction. Let $\varepsilon \in \mathscr{C}^{d+1}\left(\mathbb{R}^{d}\right)$ be a function which is symmetric under all reflections with respect to the coordinate planes and such that for all multiindices $m=\left(m_{1} \ldots m_{d}\right)$ with $|m| \leqq d+1$ we have

$$
\left|p^{m} \frac{\partial^{|m|}}{\partial p^{m}} \varepsilon(p)\right| \leqq c \varepsilon(p)
$$

for some constant $c>0$. We have to prove that if $n \geqq 0$ and $\left\{\psi_{x}\right\}_{x \in \Omega_{n}}$ is the wavelet basis constructed above and satisfying all the conditions in the theorem in Sect. 1 with $N=d+1$, then we have

$$
\sum_{x_{2} \neq x_{1}}\left|\varepsilon\left(x_{1} \mid x_{2}\right)\right| \leqq c 2^{-(d+1) n} \varepsilon\left(x_{1} \mid x_{1}\right)
$$

for all $x_{1} \in \Omega_{n}$.

Let $x_{i} \in \Omega_{n}$ and let us introduce the shorthand notations

$$
\alpha_{i}=\alpha\left(x_{i}\right), \quad s_{i}=s\left(x_{i}\right), \quad \xi_{0 i}=\xi\left(x_{i}\right), \quad p_{i}=p\left(x_{i}\right), \quad Q_{i}=Q\left(x_{i}\right)
$$

and

$$
\varepsilon_{i}=\max _{p \in Q_{i}}|\varepsilon(p)|
$$

We have

$$
\begin{aligned}
& \varepsilon\left(x_{1} \mid x_{2}\right)(2 \pi)^{-d} \int d p \varepsilon(p) \overline{\psi_{x_{1}}(p)} \hat{\psi}_{x_{2}}(p) \\
& =(2 \pi)^{-d} 2^{-\frac{d}{2}\left(s_{1}+s_{2}\right)} \int d p \varepsilon(p) \hat{\psi}_{a_{1}}\left(2^{-s_{1}} p_{1}\right) \hat{\psi}_{a_{2}}\left(2^{-s_{2}} p\right) \\
& \cdot \exp \left(i\left(\xi_{02}-\xi_{01}\right) \cdot p\right) \\
& =(2 \pi)^{-d} \varepsilon\left(p_{1}\right) \delta_{x_{1} x_{2}} \\
& \quad+(2 \pi)^{-d} 2^{-\frac{d}{2}\left(s_{1}+s_{2}\right)} \int d p\left[\varepsilon(p)-\varepsilon\left(p_{1}\right)\right] \overline{\hat{\psi}_{a_{1}}\left(2^{-s_{1}} p\right)} \hat{\psi}_{a_{2}}\left(2^{-s_{2}} p\right) \\
& \quad \cdot \exp \left(i\left(\xi_{02}-\xi_{01}\right) \cdot p\right) .
\end{aligned}
$$

Let

$$
\tilde{\varepsilon}\left(x_{1} \mid x_{2}\right) \equiv \varepsilon\left(x_{1} \mid x_{2}\right)-(2 \pi)^{-d} \varepsilon\left(p_{1}\right) \delta_{x_{1} x_{2}} .
$$


If $Q_{1} \cap Q_{2}=\emptyset$, then we have $\varepsilon\left(x_{1} \mid x_{2}\right)=0$. Moreover, for all multiindices $m=\left(m_{1} \ldots m_{d}\right)$ such that $|m|=d+1$, we have

$$
\begin{aligned}
& \prod_{j=1}^{d}\left|\xi_{02, j}-\xi_{01, j}\right|^{m_{j}}\left|\tilde{\varepsilon}\left(x_{1} \mid x_{2}\right)\right| \\
& \quad \leqq c 2^{-d s_{1}} \int_{Q_{1}} d p\left|\frac{\partial^{m}}{\partial p^{m}}\left[\varepsilon(p)-\varepsilon\left(p_{1}\right)\right] \overline{\hat{\psi}_{a_{1}}\left(2^{2-s_{1}} p\right)} \hat{\psi}_{a_{2}}\left(2^{-s_{2}} p\right)\right| \\
& \quad \leqq c 2^{-d s_{1}} \int_{Q_{1}} d p\left\{\sup _{p^{\prime} \in Q_{1}}\left|\nabla \varepsilon\left(p^{\prime}\right)\right| 2^{s_{1}-n} \cdot 2^{|m|\left(n-s_{1}\right)}\right. \\
& \left.\qquad \sum_{m_{1}+m_{2}=m} \sup _{p^{\prime} \in Q_{1}}\left|\frac{\partial^{m_{1}}}{\partial p^{m_{1}}} \varepsilon(p)^{\prime}\right| 2^{\left|m_{2}\right|\left(n-s_{1}\right)}\right\} \\
& \quad \leqq c 2^{-d s_{1}} \cdot\left|\varepsilon_{1}\right| \cdot 2^{-n+(d+1)\left(n-s_{1}\right)} \cdot 2^{-d\left(n-s_{1}\right)} \\
& \leqq c 2^{-(d+1) s_{1}}\left|\varepsilon_{1}\right|,
\end{aligned}
$$

where the constants $c$ do not depend on $n$. Hence, we have

$$
\left|\tilde{\varepsilon}\left(x_{1} \mid x_{2}\right)\right| \leqq c 2^{-(d+1) s_{1}}\left(1+\left|\xi_{02}-\xi_{01}\right|\right)^{-(d+1)} .
$$

Since there are at most $\left(2^{d}+2 d-2\right)$ possible values for $\alpha\left(x_{2}\right) \in s_{n} \times t_{d}$ such that $\tilde{\varepsilon}\left(x_{1} \mid x_{2}\right) \neq 0$, we have

$$
\sum_{x_{2} \cdot \xi_{2} \neq \xi_{1}}\left|\tilde{\varepsilon}\left(x_{1} \mid x_{2}\right)\right| \leqq c 2^{-(d+1)(n-1)}\left|\varepsilon_{1}\right|
$$

Finally, if $\xi_{1}=\xi_{2}$, we have

$$
\begin{aligned}
\left|\tilde{\varepsilon}\left(x_{1} \mid x_{2}\right)\right| & \leqq c 2^{-d s_{1}} \int_{Q_{1}} d p\left|\varepsilon(p)-\varepsilon\left(p_{1}\right)\right| \overline{\hat{\psi}_{a_{1}}\left(2^{-s_{1}} p\right)} \hat{\psi}_{a_{2}}\left(2^{-s_{2}} p\right) \\
& \leqq c 2^{-d s_{1}}\left(\sup _{p \in Q_{1}}|\nabla \varepsilon(p)|\right) 2^{(d+1)\left(s_{1}-n\right)} \\
& \leqq c\left|\varepsilon_{1}\right| 2^{-(d+1) n}
\end{aligned}
$$

The proof of the corollary in Sect. 1 is thus completed. Q.E.D.

Acknowledgement. This paper was written in 1991 during a visit to the Physics Department of Princeton University. I would like to thank Elliott Lieb for the hospitality and the US. National Science Foundation for support under grant \# PHY-8515288-A04.

\section{References}

[A] Albanese, C.: On the accuracy of the Schwinger-Englert approximation for molecules. Preprint

[FS] Fefferman, C., Seco, L.: On the ground state energy of a large atom. Bull. AMS 1990

[E] Englert, G : Semiclassical theory of atoms. Berlin, Heidelberg, New York: Springer 1988

[M] Meyer, Y.: Ondelettes. Paris: Hermann 1990 